\title{
Ordering and Non-Ideality of Air-Ionic Liquid Interfaces in Surface Second Harmonic Generation
}

\author{
Renata Costa ${ }^{a}$, Carlos M. Pereira ${ }^{a}$, A. Fernando Silva ${ }^{a}$, Pierre-François Brevet ${ }^{b}$, Emmanuel \\ Benichou*b \\ ${ }^{a}$ CIQUP - Physical Analytical Chemistry and Electrochemistry Group, Faculdade de \\ Ciências, Universidade do Porto, Departamento de Quìmica e Bioquìmica, Rua do Campo \\ Alegre, 4169-007, Porto, Portugal \\ ${ }^{b}$ Univ Lyon, Université Claude Bernard Lyon, CNRS, Institut Lumière Matière, F-69622 \\ Villeurbanne, France
}

Corresponding author:*Email : emmanuel.benichou@,univ-lyon1.fr

\section{Refractive indices of the Ils}

The refractive index of all ILs where measured with a refractometer, see Figure S1.

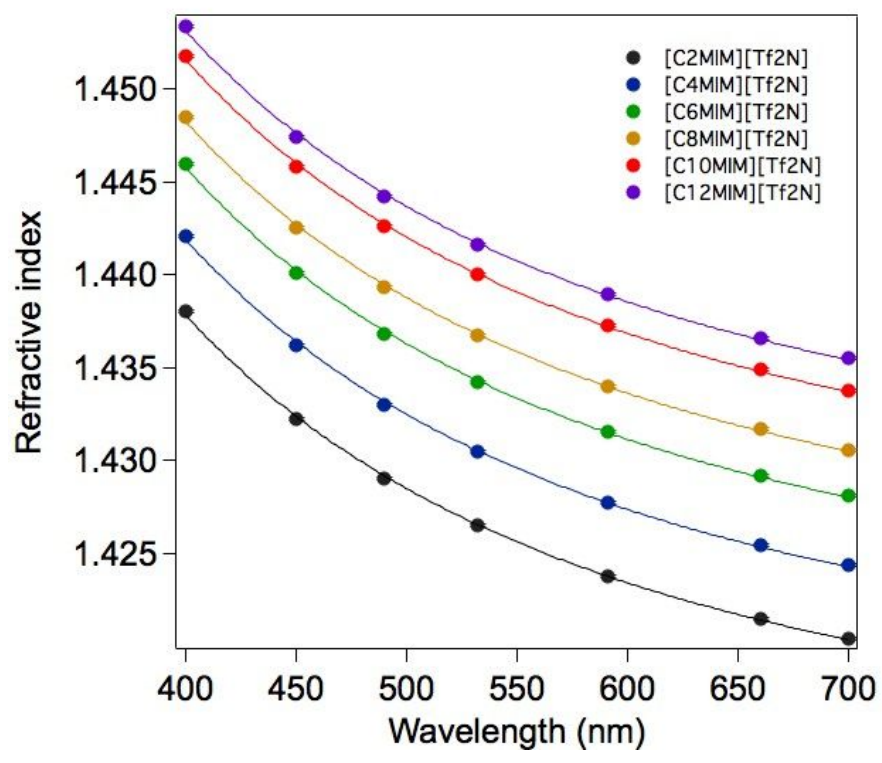

Figure S1: Refractive indices of the $\left[\mathrm{C}_{n} \mathrm{MIM}\right]\left[\mathrm{Tf}_{2} \mathrm{~N}\right]$ samples as a function of the wavelength. Solid line are adjustments of the data with Cauchy's equation (see text). 
In order to extrapolate the refractive indices of the ILs at $800 \mathrm{~nm}$, the experimental data were fitted using the Cauchy's equation:

$$
n(\lambda)=A+\frac{B}{\lambda^{2}}
$$

\section{Definition of the polarization angles}

As mentioned in the text of the article, the fundamental input beam was linearly polarized and its polarization angle $\gamma$ was selected with a rotating half-wave plate. The angle $\gamma=0$ corresponds to a p-polarized fundamental beam (in the plane of incidence) whereas $\gamma=90^{\circ}$ corresponds to a s-polarized fundamental beam (perpendicular to the plane of incidence), see Figure S2. An analyzer was used to separate the S- and P-polarized SHG intensities.

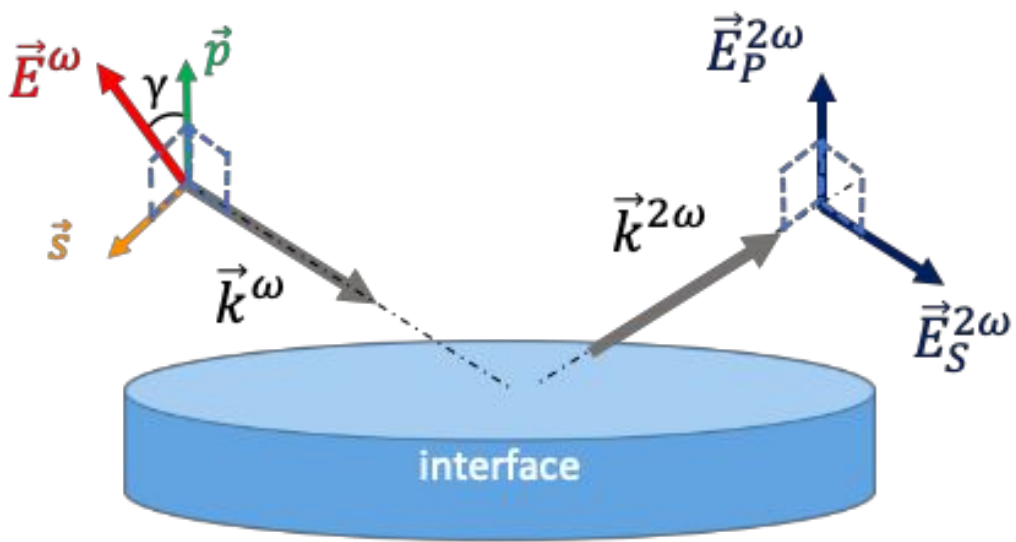

Figure S2: Definition of the polarization angles. $\vec{E}^{\omega}$ and $\vec{k}^{\omega}$ are respectively the electric field and the wave vector of the fundamental beam. The direction of this field is defined by the angle $\gamma \cdot \vec{E}^{2 \omega}$ and $\vec{k}^{2 \omega}$ are respectively the electric field and the wave vector of the harmonic light. The two directions $\mathrm{S}$ and $\mathrm{P}$ were selected in the experiment.

\section{Effect of impurities to the SHG intensity}

As mentioned in the article, the impurities in the ILs provide a background of $\mathrm{SH}$ photoluminescence which was not detrimental to the conclusions. If the global signals were 
different, the SHG intensity is not perturbed by these impurities. For example, we measured the SHG intensity from two air/IL interfaces: one is formed by $\left[\mathrm{C}_{10} \mathrm{mim}\right]\left[\mathrm{NTf}_{2}\right]$ purchased from Ioli-tec (sample 1 - purity higher than $99 \%$ ) and the other one formed by $\left[\mathrm{C}_{10} \mathrm{mim}\right]\left[\mathrm{NTf}_{2}\right]$ purchased from Solvionic (sample 2 - purity higher than 99.5\%). The following figure shows the uv-vis absorption spectra from these two ILs demonstrating clearly the presence of impurities in the Io-li-tec sample (yellowish color).
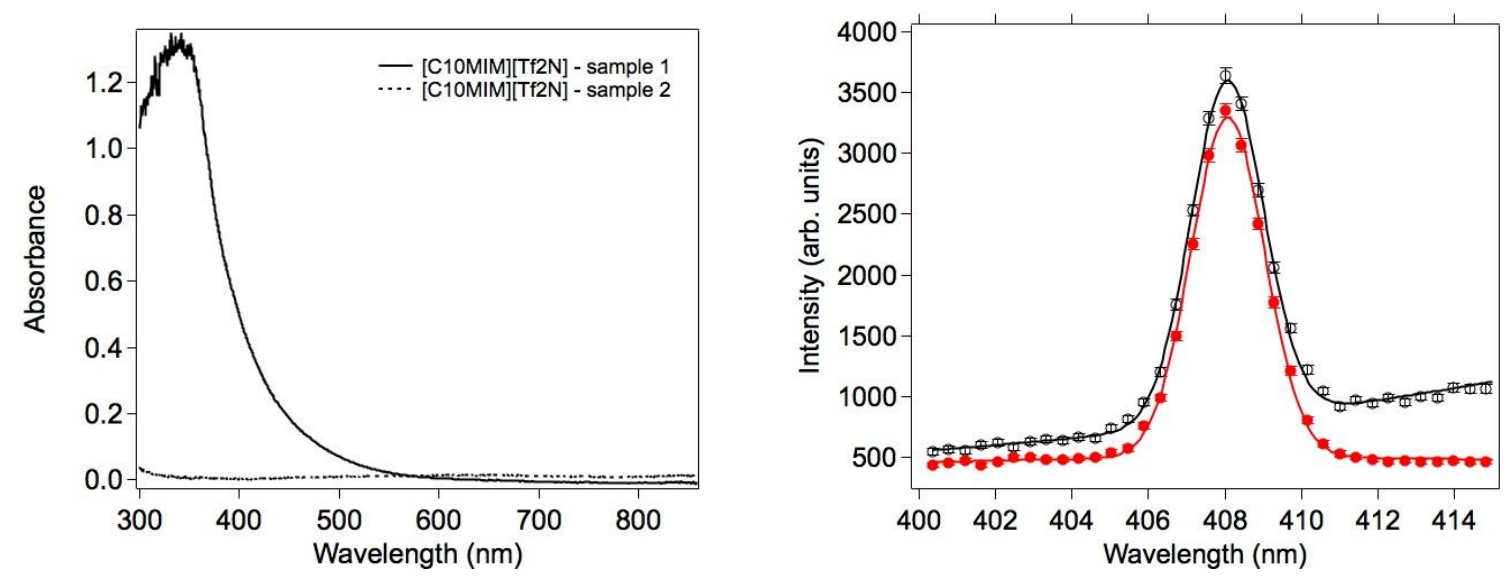

Figure S3: Left: UV-Vis absorption spectra for two samples of $\left[\mathrm{C}_{10} \mathrm{mim}\right]\left[\mathrm{NTf}_{2}\right]$ purchased from the two suppliers. Right: SHG band for the two samples of $\left[\mathrm{C}_{10} \mathrm{mim}\right]\left[\mathrm{NTf}_{2}\right]$ : sample 1 (black), sample 2 (red).

The SHG signal collected by the detection is a gaussian band centered around the harmonic frequency. The spectra obtained from the two samples are shown in Figure S3. It is clear on this figure that the presence of impurities generates a significant background on the signal. However, the height of the SHG band which is assimilated to the SHG intensity is similar for the two ILs.

\section{Susceptibility tensor elements for the air - pure ionic liquid interfaces}

Polarization resolved plots of the SHG intensity for the different air - pure ILs interfaces are reported below in Figure S4. 


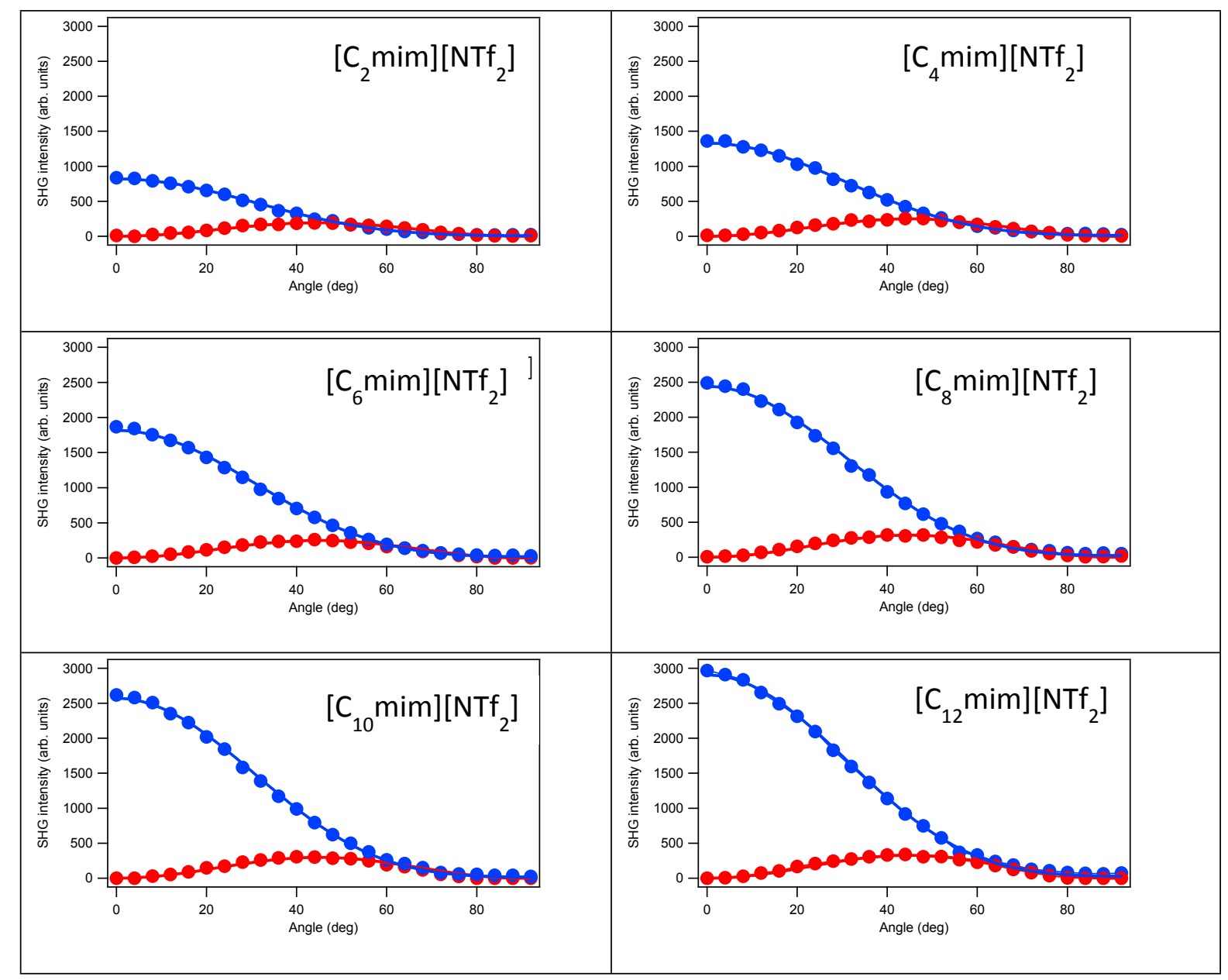

Figure S4: SHG intensity as a function of the input polarization angle for the S- (red circles), P- (blue squares) output polarization configurations obtained for the different air - pure ILs interfaces.

These plots were analyzed with the standard procedure introducing the elements of the susceptibility tensor $\chi^{(2)}$ [S1]. In the electric dipole approximation, the S-Out and P-Out SH intensities are expressed as a function of the input polarization angle $\gamma$ with the following equations : 1,2

$$
\begin{aligned}
& I_{S} \propto\left|a_{1} \chi_{x x z} \sin (2 \gamma)\right|^{2} \\
& I_{P} \propto\left|\left(a_{2} \chi_{x x z}+a_{3} \chi_{z x x}+a_{4} \chi_{z z z}\right) \cos ^{2}(\gamma)+a_{5} \chi_{z x x} \sin ^{2}(\gamma)\right|^{2}
\end{aligned}
$$

In these equations, the five $a_{i}$ parameters are coefficients depending on the incidence angle, $70^{\circ}$ in this study, and on the linear optical index of the different ILs. To calculate these 
parameters, we used the optical refractive indices reported in Table 1, both at the fundamental and at the harmonic frequency [S1].

The air - IL interface is isotropic and non-chiral. Therefore, the number of non-vanishing elements of the susceptibility tensor $\chi^{(2)}$ is only 7 with only three independent elements, namely $\chi_{X X z}, \chi_{Z X X}$, and $\chi_{z z z}$. Since the experiment was run under non-resonant conditions, these three tensor elements are assumed real value, similarly to the optical indices. The fitting procedure of the S- and P-Out polarization plots presented in Figure S3 led to the determination of these elements. However, only their relative magnitude is accessed as no absolute intensity measurements are performed. Hence, two ratios were determined, namely $\chi_{Z X X X} \chi_{X X X Z}$ and $\chi z z z / \chi_{x x z}$ and are shown below in Figure S5.
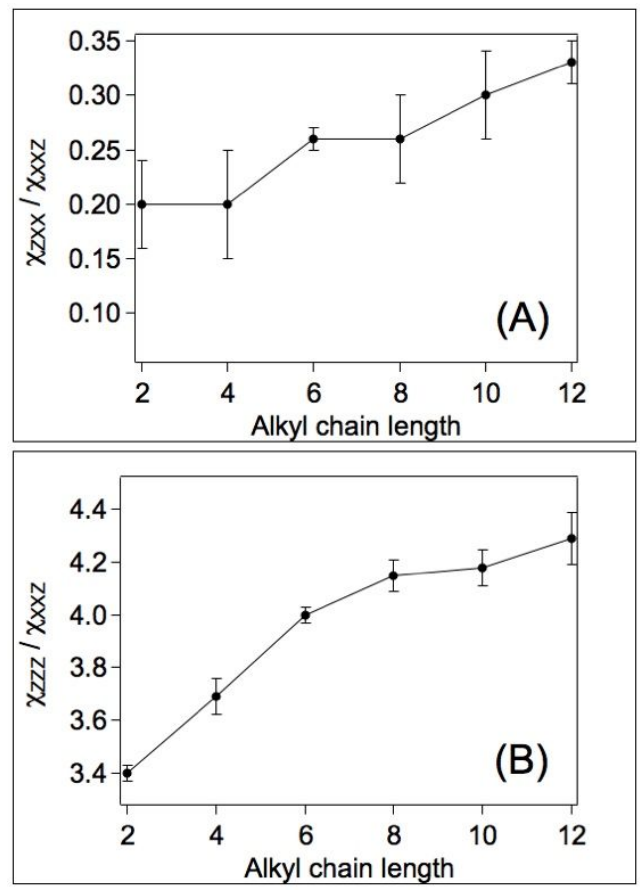

Figure S5: Susceptibility tensor ratio as a function of the IL cation alkyl chain length.

\section{Microscopic interface parameters}

Assuming that the non-linearity is dominant along the $\mathrm{z}$ axis, we consider only two non-zero microscopic tensor elements $\beta_{i j k}$ in the microscopic coordinate frame $(x, y, z)$, namely $\beta_{z z z}$ and 
$\beta_{z x x}$; In this case, it is possible to write to link the two ratios $\chi z x x / \chi x x z$ and $\chi z z z / \chi x x z$ with the $\mathrm{R}$ and D parameters as follow: ${ }^{3}$

$$
\begin{aligned}
& R=\frac{\beta_{z x x}}{\beta_{Z Z Z}}=\frac{2\left(\frac{x_{Z X X}}{x_{X Z X}}-1\right)}{\frac{x_{Z Z Z}}{x_{X Z X}}+2} \\
& D=\frac{\left\langle\cos ^{3} \theta\right\rangle}{\langle\cos \theta\rangle}=\frac{\frac{x_{Z Z Z}}{x_{X Z X}}-\frac{\chi_{Z X X}}{x_{X Z X}}+1}{\frac{x_{Z Z Z}}{x_{X Z X}}-\frac{x_{Z X X}}{x_{X Z X}}+3}
\end{aligned}
$$

The latter two ratios are provided in Figure 3 as a function of the alkyl chain length and discussed in the main text.

\section{Susceptibility tensor elements of the ILs mixture interfaces}

Susceptibility tensor elements were also determined for the air $-\left(\left[\mathrm{C}_{12} \mathrm{mim}\right]\right)_{\mathrm{x}}\left(\left[\mathrm{C}_{2} \mathrm{mim}\right]\right)_{1-\mathrm{x}}$ $\left[\mathrm{NTf}_{2}\right] \mathrm{IL}$ mixture interface from the corresponding polarization plots. These ratios are presented in Figure $\mathrm{S} 6$ as a function of the $\left[\mathrm{C}_{12} \mathrm{mim}\right]$ mixture bulk content.
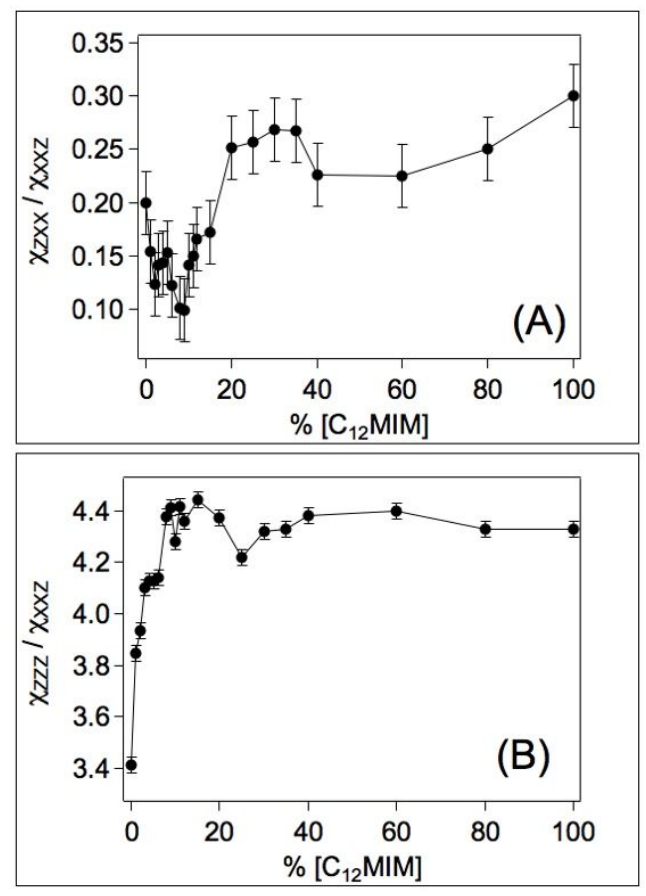

Figure S6: Susceptibility tensor element ratio as a function of the $\left[\mathrm{C}_{12} \mathrm{mim}\right]$ mixture bulk content. 


\section{References}

(1) Martin-Gassin, G.; Benichou, E.; Bachelier, G.; Russier-Antoine, I.; Jonin, C.; Brevet, P. F. Compression Induced Chirality in Dense Molecular Films at the Air-Water Interface Probed by Second Harmonic Generation. J. Phys. Chem. C 2008, 112, 12958.

(2) Bruyère, A.; Benichou, E.; Guy, L.; Bensalah-Ledoux, A.; Guy, S.; Brevet, P.-F. C. O. Reversibility of the Supramolecular Chirality of Bridged Binaphtol Derivatives at the Air-Water Interface. Opt. Mater. Express 2014, 4 (12), 2516.

(3) Zhang, W. K.; Wang, H. F.; Zheng, D. S. Quantitative Measurement and Interpretation of Optical Second Harmonic Generation From Molecular Interfaces. Phys. Chem. Chem. Phys. 2006, 8 (35), 4041-4052. 